application of MrBUMP to other classes of problem, such as complexes.

We will present the latest developments in MrBUMP, and describe a few cases studies, as well as summaries from large scale surveys.

[1] Keegan, R.M. and Winn, M.D., Acta Cryst D63, 447 (2007)

\section{MS33 O5}

Higher signal, lower noise: How to get the best data from your crystals Bram Schierbeek ${ }^{a}$, Anita Coetzee ${ }^{a}$, Martin Adam ${ }^{a}$ Matt Benning ${ }^{b}$, Roger Durst ${ }^{b},{ }^{a}$ Bruker AXS B.V., Delft, the Netherlands, ${ }^{b}$ Bruker AXS Inc., Madison WI, USA. E-mail: bram.schierbeek@bruker-axs.nl

\section{Keywords:, data collection techniques, $X$-ray detectors} SAD phasing

In a single crystal X-ray diffraction experiment in general and in Protein Crystallography in particular we aim at getting data with as high a signal-to-noise ratio as possible. Improving the signal usually involves using a more powerful source, since obtaining samples with larger diffraction volume is often not possible. The contribution of the noise however should not be underestimated. One can decrease the amount of noise in the data by a careful setup of the data collection experiment, in terms of sample handling as well as in experiment setup.

Recently we have introduced the Axiom, a new type of imaging detector for X-ray crystallography based on resistive micro-gap technology. The Åxiom is a pure digital photon-counter and thus exhibits true single-photon sensitivity with essentially zero intrinsic noise and zero frame readout dead time. This allows it to acquire both very long exposures on weakly diffracting samples without data degradation and also extremely fast exposures for time resolved experiments. It also demonstrates a very high counting rate capability of up to $10^{6}$ Xrays $/ \mathrm{mm}^{2}$-sec with a linear dynamic range of over 9 orders of magnitude (over a thousand times higher than CCD or image plate detectors). With an active area of 20 $\mathrm{cm}$ and a spatial resolution better than 100 microns the Åxiom can resolve over 400 diffraction orders. Because of the short read-out time the Axxiom can be used in shutterfree mode, where the shutter is only opened once at the start of the experiment and closed again at the end of the experiment.

This new type of detector gives opportunities for a new data collection method which can lead to a dramatic rise in signal to noise ratio, with the same amount of X-ray photons falling on the crystal. Examples of this new data collection method and its effect on signal-to-noise ratio and increased success in SAD phasing will be shown. 